\title{
Psoriasis in Pregnancy
}

\author{
Diana Sorin • Lev Pavlovsky • Michael David
}

Published online: 23 September 2012

(C) Springer Science+Business Media, LLC 2012

\begin{abstract}
Psoriasis in pregnant women requires special considerations in view of its course during pregnancy and postpartum period, the possible adverse outcomes, and the restricted basket of therapeutic measures that can be used. Most studies of pregnant psoriatic women have shown that psoriasis remained unaltered in approximately $25 \%$ of pregnancies, improved in $50 \%$, and worsened in $25 \%$. In contrast, during the 3-month postpartum period, approximately $30 \%$ remain unchanged, $10 \%$ improved, and $60 \%$ deteriorated. Psoriatic arthritis improved in the vast majority of pregnancies. Impetigo herpetiformis - a rare generalized pustular psoriasis - precipitated by pregnancy has been reported repeatedly. Moderate-to-severe psoriasis, especially when associated with comorbidities, may carry an increased risk for cesarean delivery, chronic hypertension, low birth weight, and recurrent abortions. For mild and limited disease, the use of topical mild-to-moderate-potency steroids (category C) may be used. For moderate-to-severe psoriasis, UVB phototherapy appears to be safe and effective. Anti-TNF alpha agents (category B) should not be given beyond 30 weeks of pregnancy.
\end{abstract}

Keywords Psoriasis · Impetigo herpetiformis · Pregnancy outcomes $\cdot$ NB-UVB phototherapy $\cdot$ Anti-TNF alpha

\section{Introduction}

Psoriasis is a common, chronic, inflammatory, multisystem disease with predominantly skin and joint manifestations

D. Sorin $\cdot$ L. Pavlovsky $\cdot$ M. David

Department of Dermatology, Rabin Medical Center,

Sackler School of Medicine, Tel Aviv University,

Tel Aviv, Israel

M. David $(\bowtie)$

Department of Dermatology, Rabin Medical Center,

Petach Tiqva 49100, Israel

e-mail:mdavid@post.tau.ac.il affecting approximately 1-3 \% of the population [1]. Psoriasis affects equally both sexes, and true prevalence of psoriasis in pregnant women is unknown; however, given the fact that most patients are diagnosed before age 40 years, the burden of the disease in pregnant women appears to be substantial [2].

\section{Pregnancy Effects on Psoriasis}

Psoriasis is thought to improve in $40-60 \%$ of patients during pregnancy $[3,4]$. In the study on the clinical course of psoriasis during pregnancy [5], data were collected from Psoriasis Life History Questionnaires from 91 pregnant patients with psoriasis. It was shown that the number of patients with psoriasis who improve in pregnancy is double the number of patients who worsen in pregnancy and $17.6 \%$ remained unchanged. However, the worsening of psoriasis was quite frequent during the early postpartum period. Interestingly, according to literature review [6], patients with psoriatic arthritis improved or even remitted in $80 \%$ of pregnancies. In a prospectively conducted study [7] that assessed body surface area (BSA) five times during a year in 47 pregnant patients with psoriasis (study group) and 27 nonpregnant control group patients, $55 \%$ of patients reported improvement with significant amelioration been observed from 10 to 20 weeks of gestation, $21 \%$ reported no change, and $23 \%$ reported worsening. Postpartum, $9 \%$ of patients reported improvement, $26 \%$ reported no change, and $65 \%$ reported worsening, with psoriatic BSA been increased significantly by 6 weeks postpartum. Increased estrogen levels, and especially increased ratio of estrogen to progesterone, correlated directly with improvement of psoriasis during pregnancy in this study, suggesting that estrogen might influence the shift from $T_{H} 1$ to $T_{H} 2$ immunity described in pregnancy, which results in an improvement of $\mathrm{T}_{\mathrm{H}} 1$-mediated autoimmune diseases, such as psoriasis.

Effect of Psoriasis on Pregnancy Outcome

During the past decade, major advance has been achieved in our understanding of psoriasis as multifactorial inflammatory 
immune-mediated disease and its relation to metabolic syndrome [8•,9]. Interestingly, associations with obesity and hyperglycemia, two comorbidities known to cause adverse pregnancy outcome were shown to increase with disease severity independently of other metabolic syndrome components in nonpregnant psoriasis patients [8•]. Moreover, it is well known that inflammatory and autoimmune diseases are associated with low birth weight (LBW), preterm birth (PTB), and abortions [10॰]. Data regarding the psoriasis impact on infant birth weight and other adverse pregnancy outcomes are conflicting. Recently several articles regarding this issue have been published, and we review them here. Lima et al. [11••] evaluated in retrospective cohort study the outcomes of 162 pregnancies in women with psoriasis and 501 pregnancies in women without psoriasis, using univariate and multivariate analyses and found that pregnant women with psoriasis had a higher risk for preterm birth $(<37$ gestational weeks) and low birth weight $(<2,500 \mathrm{~g})$ compared with unaffected pregnant women. Psoriasis was not associated with increased risk for cesarean delivery, preeclampsia/eclampsia, and spontaneous abortion. An impact of psoriasis severity on adverse pregnancy outcomes (PTB and LBW) was not found in this study. Yang et al. [12••] in a nationwide, population-based study compared the risk of adverse pregnancy outcomes in women with psoriasis and unaffected mothers and found that LBW was associated with psoriasis severity, which was defined as any mother who had received photochemotherapy or systemic therapy within the 2 years before the delivery. Other variables, such as small for gestational age, cesarean section, preterm birth, and preeclampsia/eclampsia, did not significantly differ compared with a control group. Mild psoriasis was not associated with excess risk of adverse birth outcomes.

On the other hand, Ben-David et al. [13] found that psoriasis carries an increased risk for cesarean delivery, chronic hypertension, and recurrent abortions. Moreover, in a retrospective, matched cohort study of 68 deliveries in 35 women with moderate-to-severe psoriasis compared with 237 deliveries in 236 women without psoriasis randomly selected after matching for age, parity, and gestational age, the psoriasis patients had a higher mean of past spontaneous and induced abortions than controls [14 ${ }^{\circ}$. The patients also had a higher percentage of pregnancy-induced hypertension and premature rupture of membranes. In multivariate analysis, moderate-to-severe psoriasis was an independent risk factor for previous spontaneous abortions, induced abortions, premature rupture of membranes (PROM), and newborn macrosomia. Independently on psoriasis impacts on pregnancy, psoriatic comorbidities should be controlled especially well during pregnancy, because it was shown that pregnant women with psoriasis may be at increased risk for adverse pregnancy outcomes due to comorbidities or other health behaviors associated with their disease [15].
What mechanism may be responsible for adverse psoriasis impact on pregnancy outcome? In psoriasis, the T-cellmediated immune response results in increased levels of certain cytokines (homocysteine, CRP, IL-6, and TNF alpha). Interestingly, it was shown that elevated maternal serum levels of CRP, TNF alpha, IL-6, and homocysteine in preeclamptic women may be associated with fetal birth weight during the early third trimester, probably due to endothelial dysfunction with resulting systemic and placental vasculopathy [16].

We conclude that psoriasis has a negative impact on pregnancy because of its direct, inflammatory effect and comorbidities. It is highly recommended to consider referring pregnant women with psoriasis to high-risk pregnancy unit.

\section{Treatment of Psoriasis During Pregnancy}

Treating psoriasis in pregnant women presents a special challenge. There are no prospective, randomized, control trials in this patient population. The Medical Board of the National Psoriasis Foundation published consensus recommendations on treatment options for psoriasis in pregnant and lactating women [17•]. We summarize these recommendations:

1. Topical treatment is the recommended first-line treatment for psoriasis in pregnant patients. Moisturizers and emollients, such as petroleum jelly, should be tried initially, because these are known not to have significant adverse effects.

Although all topical steroids are labeled as pregnancy category C by the U.S. Food and Drug Administration, the authors of the consensus recommended that low-to-moderatepotency topical corticosteroids should be attempted first, followed by high-potency topical corticosteroids only if needed in the second and third trimesters. This recommendation is based on systematic review of the safety of topical corticosteroids in pregnancy published recently [18].

Calcineurin inhibitors (Tacrolimus), although not currently approved for psoriasis, has been studied for the treatment of psoriasis in the intertriginous areas and is pregnancy category C. Salicylic acid topical treatment is not recommended at this time, because topical absorption can be substantial.

The risks of other topical agents, such as anthralin and calcipotriene, have not been reported. Thus, use of these medications is not recommended. Although the number of cases is limited, coal tar has been associated with spontaneous abortion and congenital disorders. Thus, it is not recommended for use during pregnancy.

2. Phototherapy: Second-line treatment for pregnant women is narrowband ultraviolet B (UVB) phototherapy or broadband UVB. The data for UVB phototherapy 
during pregnancy are limited [19]. To date, however, it appears to be safe and does not increase the risk of fetal abnormalities or premature delivery.

Limited cases of premature labor and fetal abnormalities have been reported in pregnant women receiving psoralen plus UVA (PUVA) photochemotherapy. Therefore, PUVA is not a recommended option during pregnancy.

3. Traditional systemic drugs: Methotrexate is an abortifacient, a teratogen, and a mutagen and therefore is contraindicated in patients who are pregnant or considering pregnancy in the short-term.

Systemic retinoids are known teratogens and therefore should be avoided during pregnancy. Systemic steroids are one of the recommended treatments for pustular psoriasis of pregnancy.

Limited information is known about the effect of cyclosporine in pregnant women with psoriasis, but it appears to confer similar risks to those in the nonpsoriatic population who typically may be prescribed lower doses. Low birth weight and prematurity when used in transplantation populations has been reported.

Cyclosporine does not appear to be a teratogen and has been used in pregnant women with successful pregnancy outcomes.

4. Biologics: There are limited human data concerning treatment of psoriasis with biologics during pregnancy, and although preliminary data appear reassuring, potential risks remain uncertain.

Experience with tumor necrosis factor (TNF) alpha inhibitors during pregnancy in psoriasis is limited to a small number of cases. Most of the data published about biologic agents in pregnancy is from rheumatoid arthritis and inflammatory bowel disease patients. All TNF blockers are classifies as category B by the U.S. Food and Drug Administration, which implies that there is no evidence of fetal risk in humans based on animal studies. Three different anti-TNF agents are now available for clinical use in psoriasis: infliximab, etanercept, and adalimumab. Two are full-length monoclonal antibodies: infliximab and adalimumab. In a recently published review [20•], the human experience with TNF inhibitors from case reports, small series, some controlled studies, and drug registries was summarized as follows: the monoclonal antibodies show increasing transplacental passage through pregnancy with concentrations in cord blood similar to or higher than maternal levels at term. On the basis of preliminary data from the Organization of Teratology and Information (OTIS) project, no significant increased risk of malformations or any consistent pattern of abnormalities have been found in exposed versus nonexposed pregnancies. One study claimed that the use of infliximab or etanercept during early pregnancy increased the rate of the congenital VACTER association (V: vertebrae malformation; A: anal anomalies; $\mathrm{C}$ : cardiac anomalies; T: tracheal problems, E: esophageal problems, R: renal anomalies), although only 1 of the 41 reported children fulfilled the criteria of the syndrome [21•]. Unfortunately, the study had neither the design nor methodology to make this statement, because it was retrospective, uncontrolled, and without knowledge of the total number of pregnancy exposures to TNF inhibitors. No other published cohort study has confirmed this observation. In utero exposure of infliximab after gestational week 30 has been shown to result in high blood levels in newborns [22]. Because TNF-alpha is known to play a role in the developing immune system, prolonged infliximab half-life in newborns could result in immunosuppression of the infant, especially for live vaccines. Fatal disseminated Bacillus Calmette-Guerin (BCG) infection occurred in a 4.5-month-old infant born to a mother with Crohn's disease who received infliximab throughout pregnancy $[23 \bullet \bullet]$. The BCG vaccine, which was given at 3 months of age, contains a live attenuated form of Mycobacterium bovis. For this reason, agents that are monoclonal antibodies, such as infliximab and adalimumab, should be discontinued before gestational week 30 . Vaccination with live viruses should be postponed in infants exposed to infliximab in utero, at least 6 months after the birth. A recent review from 2011 of registry studies and case reports involving more than 300 pregnancy outcomes in women treated with infliximab suggests that infliximab has a low fetal risk and is compatible with use during conception and the first two trimesters of pregnancy [24•].

A cohort study that included 37 pregnant women treated with etanercept for rheumatic disease before and during pregnancy revealed no major malformations. Although the numbers are limited, this study provides important population-based information to both expectant parents and prescribers $[25 \bullet \bullet]$.

Only animal studies are available for ustekinumab. Therefore, ustekinumab is not recommended during pregnancy.

\section{Impetigo Herpetiformis}

Impetigo herpetiformis is a rare pustular eruption, especially occurring in pregnancy, with the features of generalized pustular psoriasis (GPP). It has become generally accepted that impetigo herpetiformis is not a separate entity but rather a variant of pustular psoriasis in pregnancy [26]. However, there has been some controversy about this [27]. The frequent relationship with psoriasis vulgaris in the patient or her family and the worsening of pustular psoriasis just before menstruation [28] and challenge with progesterone [29] or clomiphene (clomiphene) [30] has produced pustular exacerbations in such patients are all in favor of this concept. 
Onset is usually during the last trimester of pregnancy, but may be earlier, and during the puerperium [31]. The disease tends to persist until delivery, and occasionally afterwards, may recur in subsequent pregnancies, and when taking oral contraceptives [32].

The clinical manifestations are of a GPP, usually of flexural onset and with a marked tendency to symmetry and generalization. The skin lesions may be associated with severe constitutional symptoms and death may occur, attributable to cardiac or renal failure [26].

The tongue, buccal mucosa, and even the esophagus may be involved, with circinate or erosive lesions following shortlived pustules. There is a risk of placental insufficiency leading to stillbirth, neonatal death, or fetal abnormalities [33].

Hypocalcemia often has been reported [28]. Lowered levels of serum vitamin D have been found, probably attributable to its malabsorption [34]. In principle the treatment is that of GPP - of course, with the limitations and cautions derived from pregnancy.

\section{Conclusions}

If psoriasis changes in pregnancy, it is more likely to improve than worsen, whereas during the postpartum period, it is more likely to deteriorate. Psoriasis may have a negative impact on pregnancy outcomes. NB-UVB phototherapy is the first-line treatment for moderate-to-severe psoriasis in pregnancy.

Disclosures The authors report no potential conflicts of interest relevant to this article.

\section{References}

Papers of particular interest, published recently, have been highlighted as:

- Of importance

-. Of major importance

1. Griffiths CE, Barker JN. Pathogenesis and clinical features of psoriasis. Lancet. 2007;370:263-71.

2. Tauscher AE, Fleischer AB, Phelps KC, Feldman SR. Psoriasis and pregnancy. J Cutan Med Surg. 2002;6:561-70.

3. Dunna SF, Finlay AY. Psoriasis: improvement during and worsening after pregnancy. Br J Dermatol. 1989;120:584.

4. Boyd AS, Morris LF, Phillips CM, Menter MA. Psoriasis and pregnancy: hormone and immune system interaction. Int J Dermatol. 1996;35:169-72.

5. Raychaudhuri SP, Navare T, Gross J, Raychaudhuri SK. Clinical course of psoriasis during pregnancy. Int J Dermatol. 2003;42:518-20.
6. Ostensen M. The effect of pregnancy on ankylosing spondylitis, psoriatic arthritis, and juvenile rheumatoid arthritis. Am J Reprod Immunol. 1992;28:235-7.

7. Murase JE, Chan KK, Garite TJ, Cooper DM, Weinstein GD. Hormonal effect on psoriasis in pregnancy and post partum. Arch Dermatol. 2005;141:601-6.

8. - Langan SM, Seminara NM, Shin DB, Troxel AB, Kimmel SE, Mehta NN, Margolis DJ, Gelfand JM. Prevalence of metabolic syndrome in patients with psoriasis: a population-based study in the united kingdom. J Invest Dermatol. 2012;132:556-62. This paper further establish the association of psoriasis with associations with obesity, hypertriglyceridemia, and hyperglycemia.

9. Christophers E. Comorbidities in psoriasis. Clin Dermatol. 2007;25:529-34

10. - Canti V, Castiglioni MT, Rosa S, Franchini S, Sabbadini MG, Manfredi AA, Rovere-Querini P. Pregnancy outcomes in patients with systemic autoimmunity. Autoimmunity. 2012;45:169-75. This paper discusses pregnancy outcomes in patients with various autoimmune diseases.

11. • Lima XT, Janakiraman V, Hughes MD, Kimball AB. The impact of psoriasis on pregnancy outcomes. J Invest Dermatol. 2012;132:85-91. In this recently published retrospective study, the association of psoriasis with poor pregnancy outcomes was found.

12. •• Yang YW, Chen CS, Chen YH, Lin HC. Psoriasis and pregnancy outcomes: a nationwide population-based study. J Am Acad Dermatol. 2011;64:71-7. This study found that pregnant women with severe psoriasis had an increased risk of low birth weight infants, whereas mild psoriasis was not associated with excess risk of adverse birth outcomes.

13. Ben-David G, Sheiner E, Hallak M, Levy A. Pregnancy outcome in women with psoriasis. J Reprod Med. 2008;53:183-7.

14. - Cohen-Barak E, Nachum Z, Rozenman D, Ziv M. Pregnancy outcomes in women with moderate-to-severe psoriasis. J Eur Acad Dermatol Venereol. 2011;25:1041-7. This retrospective study shows that moderate-to-severe psoriasis is associated with spontaneous and induced abortions, pregnancy-induced hypertensive diseases, premature rupture of membranes, large-for-gestational age newborns, and macrosomia.

15. Bandoli G, Johnson DL, Jones KL, Lopez Jiminez J, Salas E, Mirrasoul N, Van Voorhees AS, Chambers CD. Potentially modifiable risk factors for adverse pregnancy outcomes in women with psoriasis. Br J Dermatol. 2010;163:334-9. This prospective study shows that pregnant women with psoriasis may be at increased risk for adverse pregnancy outcomes due to comorbidities or other health behaviors associated with psoriasis.

16. Guven MA, Coskun A, Ertas IE, Aral M, Zencirci B, Oksuz H. Association of maternal serum crp, il-6, tnf-alpha, homocysteine, folic acid and vitamin b12 levels with the severity of preeclampsia and fetal birth weight. Hypertens Pregnancy. 2009;28:190-200.

17. • Bae YS, Van Voorhees AS, Hsu S, Korman NJ, Lebwohl MG, Young M, Bebo B, Kimball AB, Foundation NP: Review of treatment options for psoriasis in pregnant or lactating women: From the medical board of the national psoriasis foundation. J Am Acad Dermatol 2011. These recently published guidelines discuss imprortant issues regarding treatment of psoriasis in pregnant women.

18. Chi CC, Wang SH, Kirtschig G, Wojnarowska F. Systematic review of the safety of topical corticosteroids in pregnancy. J Am Acad Dermatol. 2010;62:694-705.

19. Vun YY, Jones B, Al-Mudhaffer M, Egan C. Generalized pustular psoriasis of pregnancy treated with narrowband uvb and topical steroids. J Am Acad Dermatol. 2006;54:S28-30.

20. - Ostensen M, Förger F. Treatment with biologics of pregnant patients with rheumatic diseases. Curr Opin Rheumatol. 2011;23: 
293-8. This review summarizes the current knowledge regarding use of biologics in pregnant women.

21. - Carter JD, Ladhani A, Ricca LR, Valeriano J, Vasey FB. A safety assessment of tumor necrosis factor antagonists during pregnancy: a review of the food and drug administration database. J Rheumatol. 2009;36:635-41. This is the first article to suggest that women exposed to tumor necrosis factor alpha antagonists during pregnancy may experience an increased risk of congenital anomalies.

22. Vasiliauskas EA, Church JA, Silverman N, Barry M, Targan SR, Dubinsky MC. Case report: evidence for transplacental transfer of maternally administered infliximab to the newborn. Clin Gastroenterol Hepatol. 2006;4:1255-8.

23. • Cheent K, Nolan J, Shariq S, Kiho L, Pal A, Arnold J. Case report: Fatal case of disseminated bcg infection in an infant born to a mother taking infliximab for crohn's disease. J Crohns Colitis. 2010;4:603-5. This paper is an alarming case of disseminated bacillus Calmette-Guérin (BCG) infection in an infant born to a mother who had received infliximab for Crohn's disease throughout pregnancy.

24. - Djokanovic N, Klieger-Grossmann C, Pupco A, Koren G. Safety of infliximab use during pregnancy. Reprod Toxicol. 2011;32:937. Comprehensive review article on safety issues of infliximab use during pregnancy.

25. •• Viktil KK, Engeland A, Furu K. Outcomes after anti-rheumatic drug use before and during pregnancy: a cohort study among 150,000 pregnant women and expectant fathers. Scand J
Rheumatol. 2012;41:196-201. In this study, none of the children whose mothers had received antirheumatic drugs were reported to be born with major malformations.

26. Roth MM. Pregnancy dermatoses: diagnosis, management and controversies. Am J Clin Dermatol. 2011;1(12):25-41.

27. Lotem M, Katzenelson V, Rotem A, et al. Impetigo herpetiformis: a variant of pustular psoriasis or a separate entity? J Am Acad Dermatol. 1989;20:338-41.

28. Gligora M, Kolacio Z. Hormonal treatment of impetigo herpetiformis. Br J ermatol. 1982;107.

29. Shelley WB. Generalized pustular psoriasis induced by potassium iodide. JAMA. 1967;201:1009-14.

30. Yasuda T, Ito M, Mizuno A, et al. Internal factors are pustular psoriasis. In: Farber EM, Cox AJ, editors. Psoriasis. Proceedings of the 2nd International Symposium. New York: Yorke Medical; 1977. p. $140-5$.

31. Katsambas A, Stavropoulus PG, Katsiboulas V, et al. Impetigo herpetiformis during the puerperium. Dermatology. 1999;198:400-2.

32. Oumeish OY, Farraj SE, Bataineh AS. Some aspects of impetigo herpetiformis. Arch Dermatol. 1982;118:103-5.

33. Sahin HG, Sahin HA, Metin A, et al. Recurrent impetigo herpetiformis in a pregnant adolescent: a report. Eur J Obstet Gynecol Reprod Biol. 2002;101:201-3.

34. Ott F, Krakowski A, Tur E, et al. Impetigo herpetiformis with lowered serum level of vitamin D and its diminished intestinal absorption. Dermatologica. 1982;164:360-5. 